\title{
Stroke integrated care pathway during COVID-19 pandemic
}

\author{
Giovanni Frisullo $^{1,2}$ (D) Antonio Giulio De Belvis ${ }^{3,4} \cdot$ Giacomo Della Marca $^{1,5} \cdot$ Carmen Angioletti $^{4} \cdot$ Paolo Calabresi $^{1,5}$
}

Received: 4 May 2020 / Accepted: 20 May 2020 / Published online: 3 June 2020

(C) Fondazione Società Italiana di Neurologia 2020

Dear Editor,

We read with a great interest the publication on "Acute stroke management pathway during Coronavirus-19 pandemic" [1] where Baracchini et al. shared their experience in the management of stroke patients in a COVID-19 Hospital. In particular, they underlined how pre-triage, a mobile $\mathrm{CT}$ unit for COVID-19 patients, and dedicated COVID-19 areas were effective measures to deal with the COVID-19 emergency. We would like to share our experience on the changes of integrated care pathway (ICP) focused on ischemic stroke patients. The Gemelli Hospital, a large university hospital in Rome, is one of the 4 hubs in the Stroke Network of Regione Lazio, serving for almost 2 million people, and is equipped with a specific ICP for the management of acute ischemic stroke that was implemented since 2013 [2]. Since March 2020, due to the outbreak of COVID-19 pandemic, the Gemelli University Hospital was identified as a COVID19 Hospital, therefore dedicated to the management of both COVID-19 and non-COVID-19 (n-COVID-19) patients. For this reason, a specific ICP, especially for time-dependent diseases, was needed in order to clearly separate the paths and the areas of COVID-19 from n-COVID patients. In particular, stroke ICP underwent several changes since the admission to the Emergency Department (ED) where all patients underwent

Giovanni Frisullo

giovanni.frisullo@policlinicogemelli.it

1 Dipartimento Scienze dell'Invecchiamento, Neurologiche, Ortopediche e della Testa-Collo, Fondazione Policlinico Universitario A. Gemelli IRCCS - UOC Neurologia, Largo A. Gemelli, 8, 00168 Rome, Italy

2 Institute of Neurology, Catholic University, Rome, Italy

3 Fondazione Policlinico Universitario A. Gemelli IRCCS, Rome, Italy

4 Section of Hygiene, Institute of Public Health, Università Cattolica del Sacro Cuore, Rome, Italy

5 Università Cattolica del Sacro Cuore, Largo Francesco Vito, 1, 00168 Rome, Italy a two-step triage. In the first step, the pre-triage just outside the ED, the risk of COVID-19 infection, was evaluated. Based on the pre-triage, the patients were classified "COVID-19" or "suspected COVID-19" (s-COVID-19) if they presented one of the following: fever $\left(\mathrm{T}>37.5^{\circ} \mathrm{C}\right)$, respiratory symptoms (cough, short breath), interstitial involvement at chest X-ray if performed before admission, or if recent contact with confirmed or probable COVID-19 patients was reported [3]. The s-COVID-19 patient undergoes nasopharyngeal swabs, taken from the nasopharynx and from each nostril. Since the result of this test need at least three hours, in the meantime, the s-COVID-19 patient is managed within the COVID-19 path. Starting from this point, the pathway of the patient with acute ischemic stroke and suspected COVID is completely separated from the n-COVID-19 one. The evaluation of sCOVID-19 patients by the stroke team of s-COVID-19 patient is carried out within a COVID-19 sub-intensive room in the ED, after wearing recommended personal protection equipment such as FFP2 respirator masks, eye protections, gowns, and gloves. If indicated by the stroke team neurologist, the patient undergoes a CT examination in a dedicated room following accesses and routes exclusively for COVID19 patients. The s-COVID-19 patients with the indication to intravenous thrombolysis (IVT), through a safe transport with bio-containment measures, carry out this treatment in the sub-intensive department, intended exclusively for patients suffering from time-dependent disease such as myocardial infarction, trauma, and stroke. A team consisting of internists, cardiologists, neurologists, and infectious disease specialists guarantees a multidisciplinary approach to sCOVID-19 stroke patients subjected to multiparametric monitoring of vital parameters. Instead, in the presence of large intracranial vessel occlusion, endovascular thrombectomy (EVT) is performed in the angiography room intended for COVID-19 patients. The s-COVID-19 patients, after performing the revascularization procedures need respiratory support, are transferred to the COVID-19 intensive care unit. Anesthesiologists, resuscitators, and intensive care specialists manage this ward, supported by stroke team neurologists. The n-COVID-19 stroke patients, on the other 
hand, follow a "clean" path that provides emergency room management within the ordinary emergency room, the execution of CT, angio-CT, perfusion CT, and possibly angiography in the "clean" sector and then transferred to the Stroke Unit in the case of stability of the vital parameters. Otherwise, the patient is transferred to the n-COVID-19 neurointensive care department (Fig. 1). This new stroke ICP meets the criteria of a "pit-stop model" where a multidisciplinary team comes together as single unit to successfully perform a complex task in a short time with minimal error. The integration of multiprofessional specialists can occur in the following settings: (1) COVID-19 sub-intensive room, where stroke team, infectious disease specialists, and resuscitators manage the patient together; (2) in the COVID19 CT room, where the patient can simultaneously perform brain and chest CT for the diagnosis of severe acute respiratory syndrome coronavirus 2 (SARS-CoV-2) pneumonia; (3) sub-intensive department dedicated to time-dependent disease, where the multidisciplinary approach allows a more appropriate management of the COVID-19 stroke patient. We believe that the main novelty of the new stroke ICP is the creation of a sub-intensive ward for time-dependent diseases in s-COVID-19 patients. In this way, a multidisciplinary approach and a multi-parameter monitoring are guaranteed to a patient more complex than the patient affected "only" by ischemic stroke, without renouncing a high specialization ensured by the presence of the stroke team. Moreover, this stroke ICP, with a definite separation between the two paths, is the model that best guarantees against possible contamination of "clean" environments, minimizing risks for nCOVID-19 patients. Finally, the creation of a multidisciplinary team in a flexible ward, able to manage all timedependent diseases, can be well suitable to the "phase two," an intermediary period between the current strict lockdown and "phase three," during which the country will begin its gradual return to normality.
Fig. 1 Stroke ICP during COVID-19 pandemic

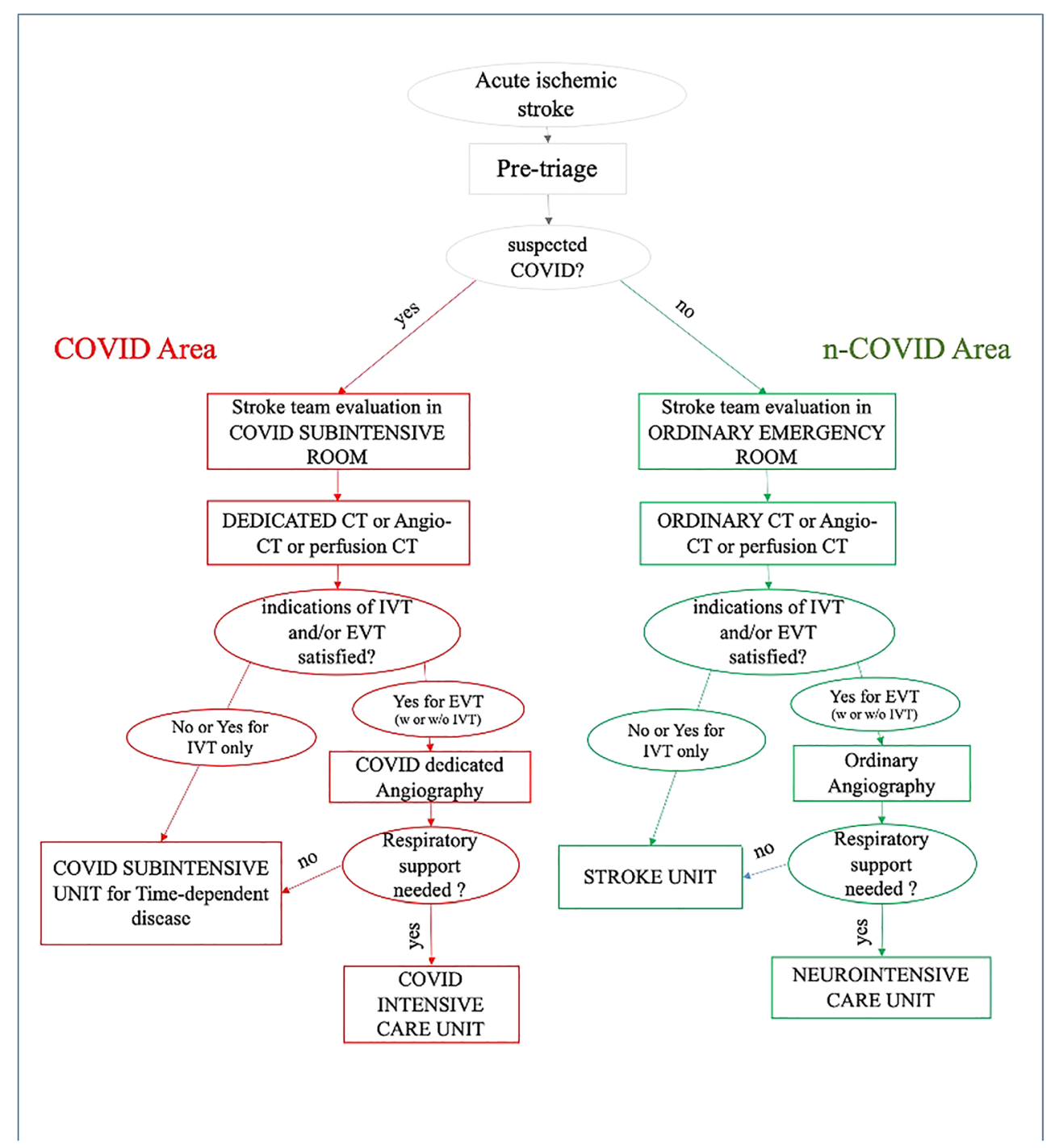




\section{Compliance with ethical standards}

Conflict of interest None.

Ethical approval None.

\section{References}

1. Baracchini C, Pieroni A, Viaro F, Cianci V, Cattelan AM, Tiberio I, Munari M, Causin F (2020) Acute stroke management pathway during Coronavirus-19 pandemic. Neurol Sci. https://doi.org/10. 1007/s10072-020-04375-9

2. Organization WH. Operational considerations for case management of COVID-19 in health facility and community. https://apps.who.int/ iris/bitstream/handle/10665/331492/WHO-2019-nCoV-HCF operations-2020.1-eng.pdf. 19 March 2019.

3. De Belvis AG, Lohmeyer FM, Barbara A, Giubbini G, Angioletti C, Frisullo G, Ricciardi W, Specchia ML (2019) Ischemic stroke: clinical pathway impact. Int J Health Care Qual Assur 32(3):588-598. https://doi.org/10.1108/IJHCQA-05-2018-0111

Publisher's note Springer Nature remains neutral with regard to jurisdictional claims in published maps and institutional affiliations. 